\title{
A Physical Activity Learning Module Improves Medical Students' Skills and Confidence for Advising Patients about Physical Activity
}

\author{
Sandra Mandic ${ }^{1}$, Hamish Wilson², Monika Clark-Grill ${ }^{2,3}$, Diana O’Neill ${ }^{4}$
}

\begin{abstract}
Affiliations: 'University of Otago, Active Living Laboratory, School of Physical Education, Sport and Exercise Sciences, Dunedin, New Zealand, ${ }^{2}$ University of Otago, Dunedin School of Medicine, Dunedin, New Zealand, ${ }^{3}$ University of Otago, Department of Primary Healthcare and General Practice, Wellington, New Zealand, ${ }^{4}$ Ministry of Health, Wellington, New Zealand
\end{abstract}

Correspondence: S. Mandic, University of Otago, School of Physical Education, Sport and Exercise Sciences, PO Box 56, Dunedin, New Zealand. E-mail: sandra.mandic@otago.ac.nz

\begin{abstract}
Physicians' knowledge, confidence, and prior training will influence physical activity (PA) counselling in general practice. This study evaluated the effects of a PA learning module on knowledge, skills, and attitudes toward PA counselling in third-year medical students. Students $(n=216$; age: $21.1 \pm 2.2$ years; 47\% males; participation rate 91\%) participated in a PA learning module that included tutorials, lectures, and experiential learning through providing health checks to adults. At baseline and four months after the intervention, students completed a paper questionnaire about their awareness of current PA guidelines, benefits of, and attitudes toward PA advising, perceived competence/importance of PA advising skills, and personal PA habits. Data were analysed using a t-test for dependent samples and Chi-square analysis. After the intervention, students reported a greater awareness of the PA guidelines (pre vs. post: $3.1 \pm 1.1$ vs. 3.8 \pm 0.9 ), the national PA initiative ( $4.2 \pm 0.9$ vs. $4.7 \pm 0.7)$, the importance of PA counselling in general practice $(4.1 \pm 0.8$ vs. $4.3 \pm 0.8$ ), and their confidence ( $3.2 \pm 0.9$ vs. $3.9 \pm 0.8)$, and perceived competence $(3.1 \pm 0.8$ vs. $3.8 \pm 0.8$, all $\mathrm{p}<0.05)$ in providing PA advice compared to baseline. In conclusion, exposure to a PA learning module increased medical students' awareness and knowledge of the current PA guidelines and improved their confidence and perceived competence in providing PA advice. However, even after the training, students on average perceived themselves to be only moderately competent in providing PA advice. Therefore, clinical training for medical students should be designed to improve students' competence and skills in PA counselling.
\end{abstract}

KEY WORDS Medical students, education, physical activity, physical activity advising, curriculum, intervention

@MJSSMontenegro

MEDICAL STUDENTS CONFIDENCE

http://mjssm.me/?sekcija=article\&artid=149

Introduction

Lack of physical activity (PA) and sedentary lifestyles among all segments of the population represent global public health problems in both developed and developing countries (World Health Organization, 2004). According to the World Health Organization, physical inactivity (lack of PA) has been identified as the fourth leading risk factor for global mortality, causing an estimated 3.2 million deaths annually (World Health Organization, 2015). Global recommendations for PA have been developed for children and adolescents, adults, and older adults (World Health Organization, 2010). According to the current PA guidelines, adults should engage in at least 150 minutes of moderate-intensity PA or at least 75 minutes of vigorous-intensity PA throughout the week, or an equivalent combination of moderate- and vigorous-intensity PA and include muscle strengthening activities on at least two days per week (Haskell et al., 2007). Additional health benefits are achieved by increasing PA above the minimum recommended levels (Haskell et al., 2007; World Health Organization, 2010). However, only one in four adults and one in five adolescents globally meet minimum PA recommendations (World Health Organization, 2015). 
Reducing physical inactivity by $10 \%$ worldwide is one of the nine global targets for non-communicable disease prevention (World Health Organization, 2013). Increasing PA is a societal, not just an individual problem, and requires a population-based, multi-sectoral, multi-disciplinary, and culturally relevant approach (World Health Organization, 2004). Consequently, PA is becoming an integral part of health-related initiatives across all segments of the population and in various settings including communities, workplaces, and schools, as well as extending into the fields of urban design, transportation and policy development. Given the key role of physicians in the public health domain, it is important to train medical students to use PA as a medical therapeutic option and to provide individualized PA advice to their patients.

Physicians can be effective in increasing patients' health-promoting behaviours, including PA (Elley, Kerse, Arroll \& Robinson, 2003; Harsha, Saywell, Thygerson \& Panozzo, 1996; Swinburn, Walter, Arroll, Tilyard \& Russell, 1998). However, less than one half of physicians screen patients for physical inactivity (Sherman \& Hershman, 1993; Walsh, Swangard, Davis \& McPhee, 1999) and less than one third of patients report receiving PA advice in the previous year (Croteau, Schofield \& McLean, 2006; Eakin, Brown, Marshall, Mummery \& Larsen, 2004; Wee, McCarthy, Davis \& Phillips, 1999). The main factors associated with the increased likelihood of physicians providing PA advice include physicians' beliefs (Sherman \& Hershman, 1993), knowledge (Rogers et al., 2006), and attitudes toward PA advising (Rogers et al., 2002), their confidence and prior training in PA advising, and their personal lifestyle (Frank, Hedgecock \& Elon, 2004; Frank, Rothenberg, Lewis \& Belodoff, 2000; Frank, Tong, Lobelo, Carrera \& Duperly, 2008).

Medical students (Connaughton, Weiler \& Connaughton, 2001; Vallance, Wylie \& MacDonald, 2009) and residents (Rogers et al., 2002) have modest competence and confidence in providing PA advice. PA also has intrinsic benefits for students in terms of their own health and wellbeing (Dyrbye, Satele \& Shanafelt, 2016). The authors of this paper recently reported that third year (pre-clinical) medical students in New Zealand were generally physically active and had a good understanding of the links between PA and health, but were lacking skills for PA advising and knowledge of specific PA guidelines for healthy individuals and clinical populations (Mandic, Wilson, Clark-Grill \& O’Neill, 2017). In addition, regularly active medical students felt more confident in providing PA advice and perceived a greater impact of PA advising on patients' quality of life (Mandic et al., 2017). Since physicians' confidence in PA-advising skills was significantly influenced by prior training (Rogers et al., 2002), it is essential to provide opportunities for medical students to develop effective skills in PA advising as a part of their undergraduate training. A recent systematic review of PA counselling in medical education curriculums reported positive changes in students' attitude towards PA, their PA counselling knowledge and skills, and their self-efficacy to provide PA advice (Dacey, Kennedy, Polak \& Phillips, 2014). The effective medical education programmes incorporated experiential learning, theoretically based frameworks, and students' personal PA habits (Dacey et al., 2014). The heterogeneity of the PA-related curriculum and weak study design of previous studies (Dacey et al., 2014) warrant further examination of the effects of the inclusion of curricula on PA advising in medical education. The current study examined the effects of an introductory learning module on PA on knowledge, skills, and attitudes toward PA advising in third-year medical students in New Zealand.

\section{Methods}

Participants

All third-year medical students at the Dunedin School of Medicine, University of Otago, (Dunedin, New Zealand, $\mathrm{n}=237$ ) were invited to participate in this study, and 216 students $(91 \%)$ gave signed consent and participated. The study was approved by the University of Otago Human Ethics Committee.

\section{Study design}

Participants completed a baseline survey in April 2009, participated in the intervention (April-May 2009) and completed the post-intervention survey in October 2009. Baseline and post-intervention surveys, were 15- to 20-minutes long and were completed on paper during tutorial classroom time.

\section{Intervention}

The PA Learning Module (intervention) consisted of three tutorials and one lecture related to PA advising and experiential learning through providing health checks to local residents. As a part of the health checks, medical students assessed the PA habits of the city residents and provided PA advice. Health checks were organized as a part of the Healthy Education Lifestyle Programme ("HELP" Day) in May 2009.

\section{Outcome Measures}

Outcome measures and measurement procedures have been described in detail elsewhere (Mandic et al., 2017) and are briefly summarized below.

Demographic characteristics. Basic demographic data collected included age, gender, ethnicity, country of origin, and self-reported height and weight. Body mass index was calculated as weight in kg divided by height in $\mathrm{cm}$ squared.

Assessment of knowledge, skills, and attitudes. Several questionnaires and items developed specifically for this study were used to assess knowledge, skills, and attitudes toward PA advising. Items were developed for this 
study to assess the awareness of current PA guidelines, publicly available resources on exercise prescription, the 'Green Prescription' initiative, and the benefits of exercise. These items were assessed using five-point Likert scales (1 [strongly disagree] to 5 [strongly agree]). The Green Prescription health initiative is a primary healthcare referral programme available in New Zealand since 1998 (Ministry of Health, 2016). Primary care physicians can refer inactive patients with stable medical conditions to local physical activity providers for free telephone follow-up, one on one, or group support to assist with lifestyle changes (Ministry of Health, 2016). In July 2009, the Green Prescription Initiative was transferred from Sport and Recreation New Zealand (SPARC) to the Ministry of Health New Zealand.

A validated Exercise and Physical Activity Competence Questionnaire (Connaughton et al., 2001) was used to assess students' perceived competence and importance of skills in PA advising. Specific skills included performing a physical examination, determining maximal heart rate, body mass index, and daily nutritional needs, calculating training heart rate and designing an exercise prescription. The questionnaire contained 12 statements using a six-point Likert-type scale (for competence: 1 [not competent] to 6 [very competent]; for importance: 1 [not important] to 6 [very important]).

The questionnaire assessing beliefs and attitudes toward personal exercise habits and PA advising was modelled on previous work (Abramson, Stein, Schaufele, Frates \& Rogan, 2000; Keats, Culos-Reed \& Courneya, 2007). Students were asked to put themselves in the role of a primary care physician and identify their potential motivations for advising patients about PA, demonstrate their knowledge of current PA recommendations, and identify other health professionals that could assist in providing PA advice (Mandic et al., 2017).

\section{Statistical analysis}

Demographic characteristics were described using descriptive statistics. Differences between baseline and follow-up assessments were examined using the paired $t$-test for continuous variables and Chi-square for categorical variables. Data are reported as mean \pm SD or frequency (percentage). A P-value less than 0.05 was considered statistically significant. Data were analysed using SPSS statistical software.

\section{Results}

Demographic characteristics

Third-year medical students ( $n=216$; age $21 \pm 2$ years; $47 \%$ male) participated in the intervention and completed both baseline and follow-up questionnaires ( $9 \%$ loss to follow-up) (Table 1).

\begin{tabular}{cc} 
TABLE 1 Demographic Characteristics & Total sample \\
& (n= 216) \\
\hline Gender $[\mathrm{n}(\%)]$ & $101(47)$ \\
Males & $115(53)$ \\
Females & $21.1 \pm 2.2$ \\
Age (years) & $172 \pm 9.6$ \\
Height (cm) & $67 \pm 10.6$ \\
Weight (kg) & $19.4 \pm 2.5$ \\
Body mass index (kg/m²) & $141(65)$ \\
Ethnicity* [n(\%)] & $14(7)$ \\
New Zealand European & $32(15)$ \\
Māori & $60(28)$ \\
Chinese & \\
Other & $111(51)$ \\
Country of origin & \\
New Zealand & $23(11)$ \\
Malaysia & $13(6)$ \\
China & $52(24)$ \\
Other & \\
\hline
\end{tabular}

Note: ${ }^{*}$ Some participants selected 2 or more ethnic groups; ${ }^{\dagger}$ Other countries of origin include England, Korea, Australia, Brunei, Taiwan, South Africa, Saudi Arabia, and others. Data were missing for 17 participants.

Awareness and knowledge of PA guidelines

The exposure to the intervention increased students' awareness of the Green Prescription Initiative in New Zealand and the PA guidelines for New Zealand adults (Table 2). The awareness of the American College of Sports Medicine guidelines for exercise in individuals with chronic medical conditions improved but remained low after the intervention. Although the majority of students were aware of the various benefits of 
PA before the exposure to the intervention, at the follow-up assessment significantly more students reported awareness of the benefits of both aerobic and resistance training as well as the health benefits and safety of exercise in individuals with chronic medical conditions.

TABLE 2 The effects of the intervention on students' knowledge of physical activity guidelines, initiatives, and benefits and attitudes towards PA advising

\begin{tabular}{|c|c|c|c|}
\hline & Baseline & Follow-up & p-value \\
\hline \multicolumn{4}{|l|}{ Physical Activity Guidelines \& Initiatives } \\
\hline I am aware of the current New Zealand guidelines for physical activity in healthy adults. & $3.10 \pm 1.07$ & $3.84 \pm 0.94$ & $<0.001$ \\
\hline $\begin{array}{l}\text { I am aware of the current American College of Sports Medicine guidelines for physical activity in } \\
\text { individuals with chronic diseases. }\end{array}$ & $1.38 \pm 0.66$ & $2.25 \pm 0.99$ & $<0.001$ \\
\hline I am aware of "Green Prescription" Initiative in New Zealand. & $4.24 \pm 0.93$ & $4.71 \pm 0.69$ & $<0.001$ \\
\hline \multicolumn{4}{|l|}{ Physical Activity Advising } \\
\hline I am confident in my ability to advise patients about physical activity. & $3.19 \pm 0.87$ & $3.92 \pm 0.76$ & $<0.001$ \\
\hline I perceive exercise advising as having a high priority in general practice. & $4.07 \pm 0.84$ & $4.28 \pm 0.77$ & 0.002 \\
\hline Exercise counselling impacts patients' quality of life. & $4.13 \pm 0.74$ & $4.26 \pm 0.74$ & 0.032 \\
\hline \multicolumn{4}{|l|}{ Benefits of Physical Activity } \\
\hline Some physical activity is better than none. & $4.84 \pm 0.46$ & $4.77 \pm 0.70$ & 0.241 \\
\hline $\begin{array}{l}\text { For most health outcomes, additional benefits occur as the amount of physical activity increases } \\
\text { through higher intensity, greater frequency, and/or longer duration. }\end{array}$ & $3.90 \pm 0.87$ & $3.95 \pm 0.87$ & 0.496 \\
\hline Both aerobic (endurance) and muscle-strengthening (resistance) physical activity are beneficial. & $4.38 \pm 0.76$ & $4.61 \pm 0.73$ & $<0.001$ \\
\hline $\begin{array}{l}\text { Health benefits from regular physical activity occur for children and adolescents, young and } \\
\text { middle-aged adults, older adults, and those in every studied racial and ethnic group. }\end{array}$ & $4.68 \pm 0.58$ & $4.73 \pm 0.68$ & 0.369 \\
\hline The benefits of physical activity far outweigh the possibility of adverse outcomes. & $4.17 \pm 0.80$ & $4.21 \pm 0.81$ & 0.487 \\
\hline Adults with chronic conditions obtain important health benefits from regular physical activity. & $4.03 \pm 0.82$ & $4.41 \pm 0.76$ & $<0.001$ \\
\hline $\begin{array}{l}\text { When adults with chronic conditions do activity according to their abilities, physical activity is } \\
\text { safe. }\end{array}$ & $4.26 \pm 0.76$ & $4.50 \pm 0.78$ & $<0.001$ \\
\hline $\begin{array}{l}\text { Older adults and people with chronic conditions and symptoms should consult their health-care } \\
\text { provider about the types and amounts of activity appropriate for them. }\end{array}$ & $4.62 \pm 0.65$ & $4.54 \pm 0.76$ & 0.197 \\
\hline
\end{tabular}

\section{PA advising: Attitudes, competence, and future intentions}

The exposure to the intervention increased students' appreciation of the importance of PA advising in general practice and the impact of PA advising on quality of life (Table 2). After the intervention, students also reported higher levels of confidence in their ability to provide PA advice (Table 3). At baseline, most medical students perceived PA advising to be important, and this perception was not significantly changed

\section{TABLE 3 The effects of the intervention on students' self-perceived competence in providing PA advice and importance of PA advising}

\section{Competence}

I feel competent to conduct a physical exam on a healthy adult to approve that person to begin a physical activity programme.

I feel competent to determine the maximum heart rate for a healthy adult.

I feel competent to determine the daily caloric and nutritional needs of a healthy adult.

I feel competent to determine the body mass index for a healthy adult.

I feel competent to calculate the aerobic training heart rate for a healthy adult.

I feel competent to design a physical activity prescription including frequency, duration, and intensity for a healthy adult

Overall perceived competence scale mean (out of 6 )

\section{Importance}

It is important to be able to conduct a physical exam on a healthy adult to approve that person to begin a physical activity programme.

It is important to be able to determine the maximum heart rate for a healthy adult.

It is important to be able to determine the daily caloric and nutritional needs of a healthy adult.

It is important to be able to determine the body mass index for a healthy adult.

$<0.001$

$2.90 \pm 1.54$

$3.53 \pm 1.41$

$<0.001$

$2.95 \pm 1.20$

$3.31 \pm 1.19$

$<0.001$

$5.83 \pm 0.42$

$5.86 \pm 0.50$

$\begin{array}{lll}5.05 \pm 1.21 & 5.09 \pm 1.08 & 0.685 \\ 4.24 \pm 1.21 & 4.35 \pm 1.21 & 0.204 \\ 4.87 \pm 1.01 & 4.68 \pm 1.16 & 0.026 \\ 5.24 \pm 0.97 & 5.23 \pm 0.95 & 0.899 \\ 4.14 \pm 1.15 & 4.23 \pm 1.18 & 0.318 \\ 4.96 \pm 1.19 & 4.75 \pm 1.17 & 0.021 \\ 4.75 \pm 0.80 & 4.72 \pm 0.80 & 0.651\end{array}$

It is important to be able to design a physical activity prescription including frequency, duration and intensity for a healthy adult. 
as a result of the intervention (Table 3). Although still only moderately competent, after the intervention students perceived increased competence in performing a physical examination, determining maximal heart rate and daily nutritional needs, calculating training heart rate, and designing an exercise programme, in comparison to the baseline (Table 3).

When students were asked to imagine themselves working as primary care physicians five years in the future, the main reasons for future PA advising in general practice (improved fitness, disease prevention, weight control and psychological benefits) remained unchanged between the baseline and follow-up assessments (Table 4). However, a greater proportion of students reported that they were more likely to provide PA advice for musculoskeletal health, physical appearance and social interaction at the follow-up assessment in comparison to the baseline (Table 4). After the intervention, a greater proportion of students reported that they would refer patients to a Green Prescription support (38\% increase) and physiotherapist (7\% increase), and a smaller proportion would refer patients to a personal trainer (11\% decrease) or exercise physiologist (5\% decrease) for more specific PA advice compared to the baseline (Table 4 ).

TABLE 4 The effects of the intervention on the reasons for providing PA advice in future and utilization of specialists for further advice

\begin{tabular}{lccc} 
& Baseline & Follow-up & p-value \\
\hline Reasons for providing PA advice $[\mathrm{n}(\%)]$ & & & \\
Cardiovascular fitness & $209(97)$ & $212(98)$ & 0.712 \\
General health/Disease prevention & $208(97)$ & $209(97)$ & 0.132 \\
Musculoskeletal health & $172(80)$ & $193(89)$ & $<0.001$ \\
Weight control & $206(95)$ & $199(92)$ & 0.145 \\
Physical appearance & $93(43)$ & $139(64)$ & $<0.001$ \\
Psychological benefits & $195(90)$ & $199(92)$ & 0.004 \\
Social interaction & $119(55)$ & $151(70)$ & 0.001 \\
Utilization of specialists for further PA advice [n(\%)] & & $174(81)$ & $<0.001$ \\
Green Prescription support & & $112(52)$ & $<0.001$ \\
Physiotherapist & $93(43)$ & $83(38)$ & $<0.001$ \\
Personal trainer & $97(45)$ & $35(16)$ & 0.012 \\
Exercise physiologist & $106(49)$ & $33(15)$ & 0.002 \\
Sports medicine doctor & $46(21)$ & $2(3)$ & 0.032 \\
Nurse / Nurse practitioner & $25(12)$ & $2(0.9)$ & 0.891 \\
Other medical doctor & $5(2)$ & $4(2)$ & 0.782 \\
Other & $2(0.9)$ & $4(2)$ & \\
\hline
\end{tabular}

Discussion

In the present study, the exposure to the PA learning module increased medical students' awareness and knowledge of the PA guidelines and improved their confidence and self-perceived competence in providing PA advice in future. However, even after the intervention, students still perceived themselves to be only moderately competent in providing PA advice. The exposure to the PA learning module broadened students' understanding of different reasons for providing PA advice in general practice and the awareness of other health professionals, particularly Green Prescription support personnel in New Zealand, for further PA advice for their patients. Background knowledge and confidence for PA advising provide a foundation for successful PA advising in general practice. These findings suggest the importance of incorporating PA advising-related curriculum in undergraduate medical degrees.

The present study shows that the exposure to the PA learning module significantly increased medical students' awareness of the PA guidelines, national PA-related programmes (i.e. Green Prescription Initiative in New Zealand), understanding of specific benefits of exercise such as the safety of exercise in clinical populations and the awareness of the other health professionals who can assist in providing further PA advice. These findings are consistent with the results of recent studies and findings from a recent systematic review on PA counselling in medical school education (Dacey et al., 2014; Jones, Brooks \& Wylie, 2013). The authors of the present study previously reported that New Zealand medical students had a good understanding of the links between exercise and health and positive attitudes towards PA advising in general practice (Mandic et al., 2017) and those outcomes were not changed considerably after the intervention in the present study. Although the intervention increased students' awareness of the PA guidelines for individuals with chronic medical conditions, students' self-perceived awareness of these guidelines remained low even after the intervention in this study. This finding is not surprising given the brief nature of this intervention, as well as the complexity of and background knowledge required for prescribing exercise for individuals with different medical conditions.

Due to the comprehensive background knowledge required for designing exercise prescription and the time constraints for PA advising in primary care practices, it is therefore essential to raise medical students' 
awareness of the other health professionals who could assist them in providing comprehensive PA advice for their patients, especially for patients with chronic medical conditions. In the present study, after the intervention, over $80 \%$ of New Zealand medical students reported that they would refer their patients to the Green Prescription support (38\% increase from baseline) and approximately half of the students would refer their patients to the physiotherapist ( $7 \%$ increase from baseline) compared to the baseline assessment whereas there was a reduction in proportion of students who would refer their patients to a personal trainer. These findings indicate that the exposure to the PA Learning Module in the undergraduate curriculum significantly increased medical students' awareness of the other competent health professionals who could assist in proving specific PA advice for their patients. As suggested previously (Mandic et al., 2017), offering medical students an opportunity to interact with different exercise professionals during their medical training may be an effective way of raising students' awareness of the role of those professionals and the support they can provide.

Although the exposure to the PA learning module increased students' self-perceived competence in providing PA advice, on average students perceived themselves to be only moderately competent in providing such advice. Several previous studies reported that medical students (Connaughton et al., 2001; Vallance et al., 2009) and resident physicians (Rogers et al., 2002) lacked competence for prescribing exercise. Since physicians' confidence in PA-advising skills was significantly influenced by prior training (Rogers et al., 2002), it is essential to provide opportunities for medical students to develop effective skills in PA advising as a part of their undergraduate training. A recent systematic review found that the inclusion of PA advising in medical school curriculum increased students' knowledge, skills, and self-efficacy to conduct PA counselling (Dacey et al., 2014). The results of the present study show that exposure to a short PA learning module consisting of one lecture, three tutorials, and one-day experiential learning increased students' self-perceived competence in providing PA advice and this increase was maintained for four months after the exposure to the intervention. However, even after the intervention, on average pre-clinical medical students perceived themselves to be only moderately competent in providing such advice. Therefore, more extensive training in PA advising may be necessary as a part of the undergraduate medical curriculum to develop medical students' skills, knowledge and confidence for providing effective PA advice in future. As suggested previously, improving medical students' knowledge of and confidence regarding PA promotion is a step forward and may increase the rates and effectiveness of physicians' PA counselling in the future (Jones et al., 2013).

The findings of this study have significant implication for future undergraduate medical curriculums. Given the effectiveness of the PA advising in general practice (Elley et al., 2003; Harsha et al., 1996; Swinburn et al., 1998), the multiple benefits of PA in healthy individuals and clinical populations (Warburton, Nicol \& Bredin, 2006), and global efforts to reduce physical inactivity by $10 \%$ worldwide (World Health Organization, 2013), it is essential to equip future physicians with the skills, and knowledge for PA advising. The present study shows that the inclusion of the PA Learning Module in undergraduate medical curriculum increases medical students' awareness of the PA guidelines, their self-perceived competence for providing PA advice to patients and awareness of the existence of other health professionals. Since increasing PA levels has multiple health benefits, taking an PA history should be a standard feature of routine medical assessment. Therefore, undergraduate medical training should include a comprehensively planned and delivered the curriculum related to PA assessment and advising. Our findings suggest that future tutorials on PA advising for medical students need to be designed to provide specific training for advising and focus on improving students' competence in providing patient-oriented exercise prescription.

\section{Study limitations}

Study limitations include the non-experimental pre-test/post-test study design without a control group and the assessment of students in one medical school. In addition, the newly developed PA Learning Module was designed to introduce PA advising to medical students and did not specifically aim to increase students' overall competence in providing comprehensive PA advice. Finally, the long-term effects of the exposure to the PA Learning Module as a part of the medical education curriculum on subsequent rates of PA advising by future physicians remain unknown. Despite these limitations, the current study was conducted on a large sample of pre-clinical medical students with a high participation rate ( $91 \%$ of the total sample). In addition to the lectures and tutorials, PA learning module in this study included an experiential learning through providing health checks and assessing PA habits of the city residents. Future studies need to examine the effects of comprehensive medical curriculum interventions focused on PA and health and PA advising on short-term outcomes (students' attitudes, knowledge, and confidence in providing patient-oriented exercise prescriptions) and long-term outcomes (the influence of undergraduate medical training on the subsequent rates of PA advising by future physicians in specialist and general practice). Future studies should use objective measures of the medical students' knowledge, skills, and competencies for PA advising and objective measures of students' PA.

\section{Conclusion}

The exposure to the PA learning module increased medical students' awareness and knowledge of the current PA guidelines and improved their confidence and perceived competence in providing PA advice. However, even after the training on average students perceived themselves to be only moderately competent in providing PA advice. Therefore, future clinical training on PA advising for medical students should be 
designed to provide specific training in PA advising focusing on improving students' competence and skills in providing patient-oriented exercise prescription as well as increasing their awareness of other healthcare professionals specializing in PA advice.

\section{Acknowledgments}

The authors would like to thank Sarah Jutel for administrative support and Sport and Recreation New Zealand (SPARC) for funding the project.

\section{Conflicts of Interest}

The authors have no conflicts of interest. Diana O'Neill worked as a Senior Health Advisor for SPARC at the time of study design and data collection.

\section{REFERENCES}

Abramson, S., Stein, J., Schaufele, M., Frates, E., \& Rogan, S. (2000). Personal exercise habits and counseling practices of primary care physicians: a national survey. Clinical Journal of Sports Medicine, 10(1), 40-48.

Connaughton, A. V., Weiler, R. M., \& Connaughton, D. P. (2001). Graduating medical students' exercise prescription competence as perceived by deans and directors of medical education in the United States: implications for Healthy People 2010. Public Health Reports, 116(3), 226-234.

Croteau, K., Schofield, G., \& McLean, G. (2006). Physical activity advice in the primary care setting: results of a population study in New Zealand. Australian and New Zealand Journal of Public Health, 30(3), 262-267.

Dacey, M. L., Kennedy, M. A., Polak, R., \& Phillips, E. M. (2014). Physical activity counseling in medical school education: a systematic review. Medical Education Online, 19(1), 24325.

Dyrbye, L. N., Satele, D., \& Shanafelt, T. D. (2017). Healthy Exercise Habits Are Associated With Lower Risk of Burnout and Higher Quality of Life Among U.S. Medical Students. Academic Medicine. 92(7):1006-1011

Eakin, E. G., Brown, W. J., Marshall, A. L., Mummery, K., \& Larsen, E. (2004). Physical activity promotion in primary care: bridging the gap between research and practice. American Journal of Preventive Medicine, 27(4), 297-303.

Elley, C. R., Kerse, N., Arroll, B., \& Robinson, E. (2003). Effectiveness of counselling patients on physical activity in general practice: cluster randomised controlled trial. British Medical Journal, 326(7393), 793.

Frank, E., Hedgecock, J., \& Elon, L. K. (2004). Personal health promotion at US medical schools: a quantitative study and qualitative description of deans' and students' perceptions. BMC Medical Education, 4(1), 29.

Frank, E., Rothenberg, R., Lewis, C., \& Belodoff, B. F. (2000). Correlates of physicians' prevention-related practices. Findings from the Women Physicians' Health Study. Archives of Family Medicine, 9(4), 359-367.

Frank, E., Tong, E., Lobelo, F., Carrera, J., \& Duperly, J. (2008). Physical activity levels and counseling practices of U.S. medical students. Medicine \& Science in Sports \& Exercise, 40(3), 413-421.

Harsha, D. M., Saywell, R. M., Jr., Thygerson, S., \& Panozzo, J. (1996). Physician factors affecting patient willingness to comply with exercise recommendations. Clinical Journal of Sport Medicine, 6(2), 112-118.

Haskell, W. L., Lee, I. M., Pate, R. R., Powell, K. E., Blair, S. N., Franklin, B. A., . . . American Heart, A. (2007). Physical activity and public health: updated recommendation for adults from the American College of Sports Medicine and the American Heart Association. Circulation, 116(9), 1081-1093.

Jones, P. R., Brooks, J. H., \& Wylie, A. (2013). Realising the potential for an Olympic legacy; teaching medical students about sport and exercise medicine and exercise prescribing. British Journal of Sports Medicine, 47(17), 1090-1094.

Keats, M. R., Culos-Reed, S. N., \& Courneya, K. S. (2007). An examination of the beliefs, attitudes and counselling practices of paediatric oncologists toward physical activity: A provincial survey. Paediatrics and Child Health, 12(4), 289-293.

Mandic, S., Wilson, H., Clark-Grill, M., \& O’Neill, D. (2017). Medical students' awareness of the links between physical activity and health. Montenegrin Journal of Sports Science and Medicine. 6(2), 5-12.

Ministry of Health. (2016). Green Prescriptions. Retrieved from http://www.health.govt.nz/our-work/ preventative-health-wellness/physical-activity/green-prescriptions

Rogers, L. Q., Bailey, J. E., Gutin, B., Johnson, K. C., Levine, M. A., Milan, F., .. Sherman, S. E. (2002). Teaching resident physicians to provide exercise counseling: a needs assessment. Academic Medicine, 77(8), 841-844.

Rogers, L. Q., Gutin, B., Humphries, M. C., Lemmon, C. R., Waller, J. L., Baranowski, T., \& Saunders, R. (2006). Evaluation of internal medicine residents as exercise role models and associations with selfreported counseling behavior, confidence, and perceived success. Teaching and Learning in Medicine, 18(3), 215-221.

Sherman, S. E., \& Hershman, W. Y. (1993). Exercise counseling: how do general internists do? Journal of General Internal Medicine, 8(5), 243-248.

Swinburn, B. A., Walter, L. G., Arroll, B., Tilyard, M. W., \& Russell, D. G. (1998). The green prescription study: a randomized controlled trial of written exercise advice provided by general practitioners. American Journal of Public Health, 88(2), 288-291.

Vallance, J. K., Wylie, M., \& MacDonald, R. (2009). Medical students' self-perceived competence and prescription of patient-centered physical activity. Preventive Medicine, 48(2), 164-166.

Walsh, J. M., Swangard, D. M., Davis, T., \& McPhee, S. J. (1999). Exercise counseling by primary care physicians 
in the era of managed care. American Journal of Preventive Medicine, 16(4), 307-313.

Warburton, D. E., Nicol, C. W., \& Bredin, S. S. (2006). Health benefits of physical activity: the evidence. Canadian Medical Association Journal, 174(6), 801-809.

Wee, C. C., McCarthy, E. P., Davis, R. B., \& Phillips, R. S. (1999). Physician counseling about exercise. JAMA, 282(16), 1583-1588.

World Health Organization. (2004). Global strategy on diet, physical activity and health. Geneva, Switzerland: WHO Press.

World Health Organization. (2010). Global Recommendations on Physical Activity for Health. Geneva, Switzerland: WHO Press.

World Health Organization. (2013). Global action plan for the prevention and control of NCDs 2013-2020. Retrieved from http://www.who.int/nmh/publications/ncd-action-plan/en/

World Health Organization. (2015). Health Topics: Physical Activity. Retrieved from http://www.who.int/ topics/physical_activity/en/ 